\title{
Evaluación del temefos y pyriproxifeno para el control de larvas de Aedes aegypti en condiciones de laboratorio
}

\author{
María Pérez ${ }^{1, a}$
}

RESUMEN

Objetivo: Evaluar la eficacia del temefos frente al pyriproxifeno a diferentes dosis $(0.01,0.02,0.03,0.04$ y $0.05 \mathrm{ppb})$ para el control de larvas de Aedes aegypti en condiciones de laboratorio.

Materiales y métodos: Estudio experimental con grupo de control, que incorporó a 2000 larvas de Aedes aegypti provenientes de la jurisdicción de Collique III Zona, Comas - Perú, y la cepa Rockefeller como control susceptible. Se determinó la diferencia en tiempo de inicio de la acción larvicida; así mismo, se evaluó el porcentaje de sobrevivencia y mortalidad a diferentes dosis $(0.01,0.02,0.03,0.04$ y $0.05 \mathrm{ppb})$ del temefos vs. pyriproxifeno a través de la metodología recomendada por la Organización Mundial de la Salud (OMS) en condiciones de laboratorio.

Resultados: Según los resultados obtenidos, las larvas de la cepa Collique III Zona expuestas al temefos mostraron un promedio de mortalidad de $68.4 \%$, a diferencia del ensayo con pyriproxifeno, cuyo promedio de mortalidad fue de $0.8 \%$. Con relación a la cepa Rockefeller vs. temefos, el promedio de mortalidad fue de $100 \%$, a diferencia de la cepa Rockefeller y pyriproxifeno que fue de $0.8 \%$. Esto indica que temefos tiene un efecto larvicida en menor tiempo para el control larvario de Aedes aegypti.

Conclusiones: La población de larvas de Aedes aegypti mostró susceptibilidad al insecticida temefos. Una única aplicación de temefos a la dosis de 0.04 ppm mostró una mortalidad del $92 \%$ en las larvas. Con el regulador de crecimiento (pyriproxifeno), encontramos valores de mortalidad bastante bajos en la cepa Collique y cepa Rockefeller.

Palabras clave: Dengue; Aedes aegypti; temefos; pyriproxifeno (Fuente: DeSC BIREME).

\section{Evaluation of temefos and pyriproxyfen to control Aedes aegypti larvae in laboratory conditions}

\begin{abstract}
Objective: To evaluate the efficacy of temefos versus pyriproxyfen at different doses $(0.01,0.02,0.03,0.04$ and $0.05 \mathrm{ppb})$ to control Aedes aegypti larvae in laboratory conditions.

Materials and methods: An experimental study with control group which included 2,000 Aedes aegypti larvae from the Collique III Zona jurisdiction in Comas, Peru, and the Rockefeller strain as susceptible control. The difference in the time of onset of the larvicidal effect was established. Additionally, the survival and mortality rates with temefos versus pyriproxyfen were evaluated at different doses $(0.01,0.02,0.03,0.04$ and $0.05 \mathrm{ppb})$ in laboratory conditions using the methodology recommended by the World Health Organization (WHO).

Results: Based on the results, the Collique III Zona strain larvae exposed to temefos showed an average mortality rate of $68.4 \%$, as opposed to the average mortality rate in the pyriproxyfen assay, which was $0.8 \%$. Regarding the Rockefeller strain exposed to temefos, the average mortality rate was $100 \%$, as opposed to the average mortality rate in the pyriproxyfen assay, which was $0.8 \%$. This indicates that temefos has a faster larvicidal effect to control Aedes aegypti larvae.

Conclusions: The Aedes aegypti larvae population was susceptible to insecticide temefos. A single application of temefos at $0.04 \mathrm{ppm}$ showed a mortality rate of $92 \%$ in the larvae. With insect growth regulator pyriproxyfen, very low mortality rates were found in the Collique and Rockefeller strains.
\end{abstract}

Keywords: Dengue; Aedes; temefos; pyriproxyfen (Source: MeSH NLM).

1. Biólogo, Magíster en Ciencias de los alimentos.

a. Ministerio de Salud. Lima, Perú. 


\section{INTRODUCCIÓN}

El dengue es una enfermedad viral de prevalencia global que ha aumentado dramáticamente en las últimas décadas, transmitida por mosquitos del género Aedes, principalmente Aedes aegypti ${ }^{(1)}$. Así mismo es transmisor de la Fiebre Amarilla, Chikungunya y Virus Zika estas enfermedades representan un grave problema de salud pública y pueden afectar negativamente la economía de los países en la región ${ }^{(2)}$. El ciclo biológico del mosquito consiste en cuatro etapas: huevo, larva, pupa y adulto. Los huevos, preferentemente son depositados en recipientes artificiales domiciliares y peridomiciliares, en condiciones ideales de temperatura la eclosión ocurre en un par de días. Los estadíos larvarios se desarrollan en hábitats acuáticos. Después ocurre una segunda fase acuática de pupa, en esta fase la alimentación se detiene y después de uno o dos días emerge el adulto, que es un mosquito volador y pequeño ${ }^{(3)}$.

Las hembras poseen una probóscis larga, adaptada para succionar sangre a través de la piel. Sin embargo los machos presentan una probóscis adaptada para succionar jugos de plantas y otras fuentes de azúcar. Además de ello las hembras dependen de sangre para obtener la energía necesaria para la producción de los huevos, siendo las horas con mayor actividad alimenticia entre las 6:00 am a 8:00 am y de 4:00 pm a 7:00 pm ${ }^{(4)}$.

El virus del dengue es transmitido por la picadura del mosquito hembra adulto, que a su vez es infectado al picar a un sujeto enfermo, vive principalmente en regiones tropicales, limitadas entre los $35^{\circ}$ de latitud norte y $35^{\circ}$ de latitud sur, pero no se encuentra habitualmente por encima de los 1000 metros de altura ${ }^{(5)}$.

Diversos factores han sido relacionados con la reemergencia de esta enfermedad, particularmente, el crecimiento poblacional, la urbanización no planificada, insuficiente abastecimiento de agua, así como la gestión incorrecta de alcantarillado y el manejo de los residuos sólidos. También han sido importantes el incremento de los viajes aéreos, la migración y el deterioro de los programas de prevención y control ${ }^{(6)}$.

Actualmente las enfermedades transmitidas por Aedes aegypti se mantienen en áreas tropicales del Tercer Mundo y están distribuidas en áreas urbanas de Asia, el Pacífico, África, América Latina y el Caribe ${ }^{(7)}$.

En las últimas cinco décadas, la incidencia se ha incrementado 30 veces documentándose en arias previamente no afectadas, cada año surgen cientos de miles de casos de dengue grave, con aproximadamente 20000 muertes. Anualmente se pierden en el mundo 264 años de vida ajustados por discapacidad por millón de habitantes, con un costo estimado de casos ambulatorios y hospitalizados de USD 514-1394, afectando mayormente a las poblaciones más pobres. Así mismo el dengue en el Perú, los primeros reportes de brotes de un síndrome febril compatible con dengue clásico en el país fueron descritos en 1700, 1818, 1850 y 1876, aunque no se tuvo confirmación por Laboratorio. La reemergencia del dengue en el Perú en el siglo XX está ligado a la reintroducción del Aedes aegypti en 1984, reportándose la primera epidemia explosiva en 1990 por el serotipo DENV-1, en las principales ciudades de la Amazonía y en la actualidad en casi todas las áreas del país con casos de dengue y la circulación de los cuatro serotipos DENV-1, DENV-2, DENV-3 y DENV-4. Convirtiéndose así en una enfermedad de importancia para la salud pública ${ }^{(8)}$.

El control del vector del dengue es aún la única medida disponible para disminuir la emergencia y trasmisión de esta enfermedad viral. El control integrado de vectores (CIV) es la estrategia más importante que se debe considerar a la hora de decidir o ejecutar las acciones de control de Aedes agypti. La estrategia de CIV incluye la utilización de diferentes métodos, saneamiento ambiental, control químico, control biológico y la introducción de métodos genéticos más modernos. A pesar de que se implementen varias medidas de control, en ocasiones no es suficiente, dando paso a la ocurrencia de brotes o epidemias de dengue; resultando el control químico la medida más eficaz para controlar la transmisión de dengue. El insecticida órganofosforado Temefos ha sido el larvicida más utilizado en Cuba por el Programa de Control de Aedes. aegypti, el cual se ha aplicado en formulaciones granuladas a una concentración de $1 \mathrm{ppm}$. El primer diagnóstico de resistencia a Temefos en Cuba fue en Santiago de Cuba, con la ocurrencia del brote de dengue en este municipio y posteriormente, en la ciudad de la Habana. De aquí la necesidad de evaluar otros métodos de control que sean eficaces para control de poblaciones de Aedes. aegypti, que hayan desarrollado resistencia a Temefos. El uso del Pyriproxifeno, regulador de crecimiento, constituye una alternativa como parte del CIV. Es un análogo de la hormona juvenil que inhibe la producción de huevos y la metamorfosis, su poder de acción es principalmente contra los insectos chupadores y tiene poco efecto en los himenópteros; también es efectivo contra las larvas de mosquitos y aunque puede ser tóxico para los organismos acuáticos, la dosis diferencial entre la mayoría de estos y las pupas de los mosquitos es tan grande, que lo convierte en una buena elección para el control de mosquitos en ambientes sensibles ${ }^{(9)}$.

En Lima se ha usado sistemáticamente el larvicida Temefos para el tratamiento focal de estadios inmaduros de Aedes aegypti desde su reaparición en el año 2000; en el 2005 se reportó que era susceptible a dosis de $0,025 \mathrm{mg} / \mathrm{dL}$, situación similar al reporte en Trujillo. Sin embargo, el uso constante de este insecticida produciría una presión de selección, ocasionando la aparición de poblaciones resistentes como se reporta para Cuba y Brasil ${ }^{(10)}$.

El presente estudio pretende evaluar la eficacia del Temefos vs Pyriproxifeno en larvas de Aedes aegypti procedentes de la Jurisdicción de Collique III zona - Comas, a las diferentes dosis de $0.01,0.02,0.03,0.04$ y 0.05 ppb con el fin de proporcionar información relevante respecto a la eficacia y contribuir de esta forma con las estrategias 
de prevención y control de las enfermedades transmitidas por el vector Aedes aegypti.

\section{MATERIALES Y MÉTODOS}

\section{Selección del área de estudio}

La jurisdicción de Collique III Zona - Comas, fue elegida por la importancia epidemiológica permanente de brotes autóctonos de dengue, zona de fácil acceso, situación de orden público manejable y reporte de altos índices aédicos.

\section{Técnicas de recolección de muestra}

\section{Recolección de larvas y pupas de Aedes aegypti en campo}

Se realizó visitas domiciliarias aleatoriamente a la jurisdicción de Collique III zona previo consentimiento informado. Se inspeccionaron criaderos potenciales de Aedes aegypti como tanques bajos de concreto, cilindros y otros recipientes que son criaderos potenciales de larvas, estas fueron recolectadas con la ayuda de un cucharón y gotero, se trasvasaron a bandejas de plástico con agua del mismo recipiente, este se tapó herméticamente para evitar el vertimiento del agua y así fueron transportadas al Laboratorio de Entomología del Instituto de Medicina Tropical de la Universidad Nacional Mayor de San Marcos ${ }^{(11)}$.

\section{Obtención de primera generación de Aedes aegypti en laboratorio}

Las larvas colectadas en campo se transfirieron a bandejas plásticas de $30,5 \times 20 \times 10 \mathrm{~cm}$ con un volumen considerable de agua reposada alimentándolas diariamente con alimento balanceado conejina $1 \mathrm{gr}$, hasta que alcanzaron el estadio de pupa. Posteriormente se separaron en frascos de $500 \mathrm{ml}$ de capacidad llenos con $250 \mathrm{ml}$ de agua declorada hasta la emergencia de adulto; estos se liberaron en jaulas entomológicas de $30,5 \times 30,5 \times 30,5 \mathrm{~cm}$. Los mosquitos machos se alimentaron con solución azucarada al $5 \%$ y las hembras se alimentaron cada tercer día con sangre de pollo.

Para la ovipostura, se introdujo en la jaula un vaso desechable de color oscuro de $500 \mathrm{ml}$ que contenía en su interior toallas de papel absorbente y un tercio de volumen de agua reposada. Estas toallas se retiraron cada tercer día rotulándose con el nombre, procedencia y fecha. Los huevos se mantuvieron en cámara húmeda durante las siguientes 48 horas para su secado y posteriormente almacenamiento en recipientes de plásticos herméticos. Al contar con un número suficiente de huevos para el experimento, las toallas de papel se sumergieron en bandejas con agua para obtener el material entomológico a ser evaluado correspondiente a la generación F1 (cepa pura). El material entomológico se mantuvo bajo condiciones controladas en el laboratorio con una temperatura de $27+/-2^{\circ} \mathrm{C}$, humedad relativa entre $65 \%$ y
$81 \%$. Además de ello simultáneamente se realizó el mismo procedimiento con la Cepa Rockefeller de referencia de Aedes aegypti susceptible a los insecticidas, para luego ser enfrentadas a las diferentes dosis de Temefos y Pyriproxifeno ${ }^{(12)}$.

Preparación de las soluciones de Temefos y Pyriproxifeno

Se preparó cinco dosis $(0.01,0.02,0.03,0.04$ y 0.05 ppm) de y Temefos y Pyriproxifeno según la metodología de la Organización Mundial de la Salud (OMS) ${ }^{(13)}$.

\section{Bioensayos con Temefos}

Los bioensayos se realizaron según la metodología de la OMS (14). Se colocaron 5 réplicas por cada dosis del insecticida con 20 larvas del III y IV estadío. Todas las soluciones se ajustaron a un volumen final de 250 a $300 \mathrm{ml}$. La lectura se realizó a partir de la exposición y durante el tiempo que dure el bioensayo, es decir mueran todos los especímenes en estudio, similar procedimiento se realizó con la cepa Rockefeller. Así mismo para la recolección de datos se diseñó una ficha. Los datos se analizaron mediante la (prueba no paramétrica $U$ de Mann Whitney, $p=0,008$, $p<0,05)$.

\section{Bioensayos con Pyriproxifeno}

Los bioensayos se realizaron según la metodología de la OMS. Se colocaron 5 réplicas por cada dosis del Pyriproxifeno, con 20 larvas de III y IV estadio. Todas las soluciones se ajustaron a un volumen final de $250 \mathrm{ml} \mathrm{a}$ $300 \mathrm{ml}$. La lectura se realizó a partir de la exposición y durante el tiempo que dure el bioensayo, es decir mueran todos los especímenes en estudio, similar procedimiento se realizó con la cepa Rockefeller. Así mismo para la recolección de datos se diseñó una ficha. Los datos se analizaron mediante la (prueba no paramétrica $U$ de Mann Whitney, $p=0,008, p<0,05)$.

\section{RESULTADOS}

Los resultados de los bioensayos realizados de acuerdo a los protocolos de la Organización Mundial de la Salud (OMS) muestran el promedio de mortalidad de larvas expuestas a Temefos en un $68.4 \%$, sin embargo el promedio de mortalidad de larvas expuestas al Pyriproxifeno de $0.8 \%$. Sin embargo el porcentaje de mortalidad en los bioensayos realizados con Cepa Rockefeller vs Temefos mostraron una mortalidad de $100 \%$, en relación al ensayo con Cepa Rockefeller vs Pyriproxifeno fue de $4.2 \%$. Se analizaron varias dosis de Temefos vs Pyriproxifeno a diferentes dosis de $0.01,0.02,0.03,0.04$ y 0.05 ppm determinándose la dosis de $0.04 \mathrm{ppm}$ una mortalidad de $92 \%$ lo que demuestra que esta dosis debería ser utilizada para el control de larvas del Aedes aegypti, contribuyendo así a la disminución del vector del dengue. 


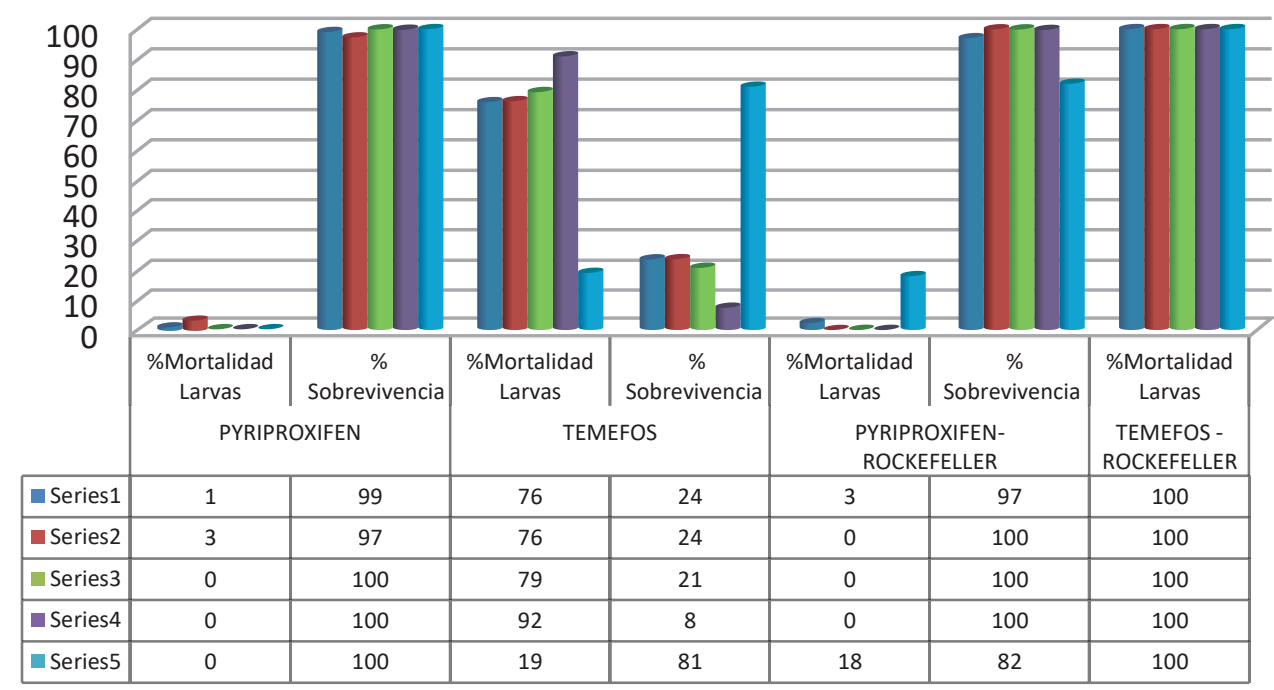

Figura 1. Porcentaje de mortalidad y sobrevivencia de larvas de Aedes aegypti - Rockefeller expuestas por réplica a Temefos vs Pyriproxifeno

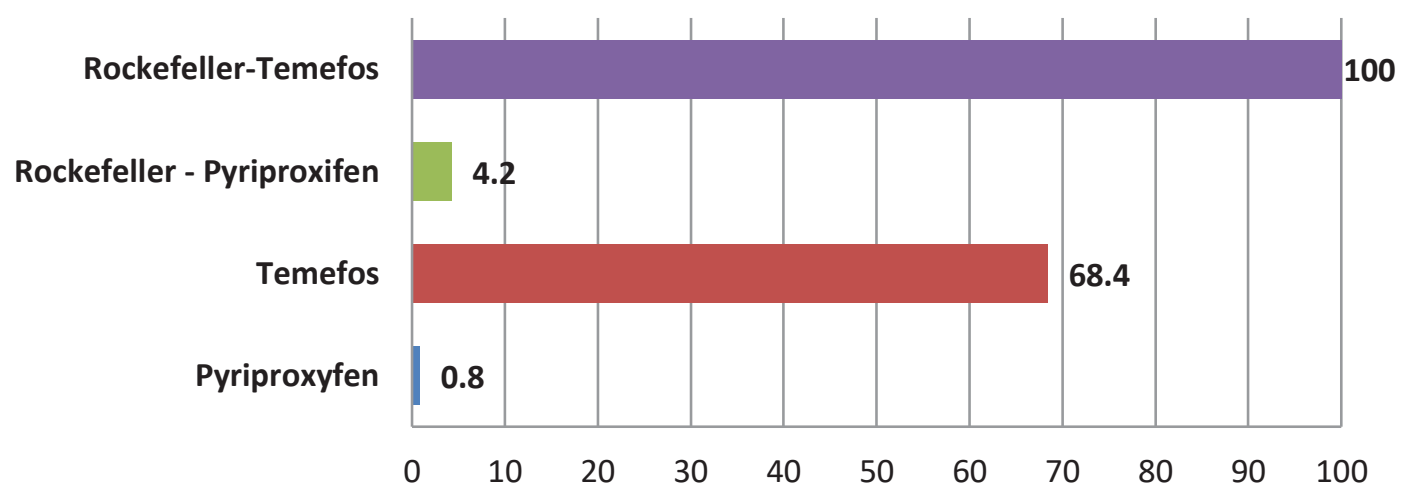

Figura 2. Promedio de mortalidad y sobrevivencia de larvas de Aedes aegypti-Cepa Rockefeller expuestas a Temefos y Pyriproxifeno

Los resultados obtenidos en el presente trabajo indican que el insecticida Temefos puede seguir siendo utilizado en los programas de control vectorial del Aedes aegypti, a la vez se hace necesario realizar vigilancia continua de los niveles de resistencia de poblaciones naturales, pues el Aedes aegypti podría convertirse en una amenaza para las operaciones de control químico adecuado dado que el vector se está adaptando a las diversas condiciones del ambiente.

\section{DISCUSIÓN}

En Perú, el Temefos es utilizado por el Ministerio de Salud, para el control vectorial de Aedes aegypti en las campañas contra el dengue y otras enfermedades transmitidas por este vector. Así, mismo los lugares con altos índices de infestación larvaria que constituyen zonas mayormente afectadas por las últimas epidemias de dengue y brotes de fiebre Chikungunya reciben una intervención mucho mayor $y$ en ese contexto es importante mencionar que el uso contante del mismo larvicida genera presión de selección, favoreciendo la aparición de poblaciones resistentes. Según Camargo et al, señalaron que la condición fundamental que favorece la infestación de mosquitos en el área urbana es la dificultad en el suministro de agua potable, lo que obliga a la población a mantener numerosos depósitos para su recolección y almacenamiento ${ }^{(15)}$.

De esta manera, el estudio realizado demostró susceptibilidad al Temefos en la población de larvas de Aedes aegypti procedentes de la jurisdicción de Collique III zona - Comas. Se demostró que el porcentaje de mortalidad es superior a los resultados obtenidos con Piryproxifeno mostrando así un alto nivel de susceptibilidad para el Temefos, este comportamiento poblacional debe ser considerado para seguir siendo utilizado para el control del vector Aedes aegypti. Según Rodríguez et al, refirieron que el tratamiento focal incluye la eliminación o modificación de los criaderos, con participación de la comunidad, y la aplicación de larvicidas en aquellos depósitos donde no es posible destruirlo ${ }^{(16)}$. 
No obstante, un estudio realizado en Argentina demostró que entre los años 2006 / 2007, se habían detectado valores de resistencia insipiente a Temefos en larvas de Aedes aegypti colectadas en dicha ciudad, en ese sentido, este estudio discrepa con los resultados de Argentina, teniendo en cuenta que los valores de mortalidad fueron superiores para el Temefos y en relación al Pyriproxifeno los resultados de mortalidad fueron muy bajas, sin embargo no se puede descartar la posibilidad de una introducción pasiva de haplotipos resistentes hacia nuestro país, mas aun considerando que el Perú es un país fronterizo con intenso tráfico comercial y migración de personas que pueden contribuir a la expansión de genes involucrados con la resistencia a los organofosforados de uso frecuente. Según Reiner et al, el Temefos es un órganofosforado que actúa inhibiendo la acetilcolinesterasa en las unidades sinápticas por fosforilación. Generalmente actúan en las larvas de los primeros estadios, hasta el tercero principalmente, cuando estas ingieren alimentos, pero no actúa en el estadio de pupa ${ }^{(17)}$.

Por otra parte el uso del Temefos para el control químico de larvas de mosquitos por largos periodos de tiempo contribuye a un proceso de selección de individuos resistentes, por lo cual sería inminente la aparición de resistencia a este larvicida como ya fue demostrado en otros estudio recientes realizado con cepas de Aedes aegypti de Asunción, en el cual las poblaciones presentaron valores de resistencia insipiente. Según Sharmaet et al, realizaron un estudio en la India, donde se ha demostrado que tanto Aedes aegypti como Aedes albopictus resultaron susceptibles a los órganofosforados más utilizados en los programas de control, entre estos a Temefos, que demuestra una similitud con los resultados obtenido en la presente investigación ${ }^{(18)}$. En un estudio realizado por Chávez et al, encontraron susceptibilidad a temefos en todas las poblaciones de Aedes aegypti estudiadas, al presentar valores de factor de resistencia menores. Similares a los resultados obtenidos en el trabajo de investigación ${ }^{(19)}$. Recientemente la Organización Mundial de la Salud (OMS), ha brindado como otras alternativas para el tratamiento focal en aguas de consumo humano el uso del Pyriproxifeno a dosis hasta de $0.01 \mathrm{mg}$, por ser ovicida, larvicida y pupicida para ser utilizado en el control de larvas de Aedes aegypti, este viene siendo usado en tanques de almacenamiento de agua en lquitos - Perú, que fue aplicado a una dosis de $0.012 \mathrm{ppm}$ dando resultados relativamente considerables.

La población de larvas de Aedes aegypti de la jurisdicción de Collique III zona - Comas, mostró una mayor eficacia al insecticida Temefos, observándose una mortalidad de $68.4 \%$. Así mismo la eficacia del Temefos frente a cepa Rockfeller fue del $100 \%$, también es necesario mencionar que a dosis de $0.04 \mathrm{ppm}$ hubo un $92 \%$ de mortalidad sin embargo con el Pyriproxifeno expuesto con la cepa Collique III zona - Comas la mortalidad fue $0.8 \%$. Así mismo la eficacia del Pyriproxifeno vs cepa Rockefeller fue de $4.2 \%$. Los resultados obtenidos en el presente trabajo indican que el insecticida Temefos puede seguir siendo usado en los programas de control vectorial del Aedes aegypti como larvicida. A la vez, es necesario realizar monitoreo continuo de los niveles de resistencia en poblaciones de Aedes aegypti y así hacer un buen uso del Temefos en el control de este vector en el Perú.

En conclusión, la población de larvas de Aedes aegypti evaluada mostro susceptibilidad al insecticida Temefos, en un $68.4 \%$, confirmando la eficacia del producto, en relación al Pyriproxifeno la mortalidad fue de $0.8 \%$.Así mismo se determinó la dosis de $0.04 \mathrm{ppm}$ con una mortalidad de $92 \%$ en larvas de Aedes aegypti.

\section{RECOMENDACIONES}

Se requiere vigilancia entomológica periódica para monitorear la evolución de la posible resistencia de larvas de Aedes aegypti al Temefos.

En investigaciones futuras se debe realizar estudios en condiciones en campo con Pyriproxifeno para determinar la dosis ideal y el efecto residual a ser utilizado como una alternativa en el Programa de Control de Vigilancia del vector en el Perú.

\section{REFERENCIAS BIBLIOGRÁFICAS}

1. Molina de Fernández D, Bastidas D, Figueroa L. Malation vs. Aedes aegypti (Linnaeus, Diptera: Culicidae) de diferentes regiones de Venezuela, Boletín de Malariología y Salud Ambiental. 2013; 53(1): 46-55.

2. Ferreira M, dos Santos L, de Melo C, Pereira J, Gonzalez N. Perfil de susceptibilidad a Temefos en poblaciones de Aedes aegypti (Diptera: Culicidae) de Ciudad del Este - Alto Paraná, Paraguay Mem, Inst, Investig, Cienc. Salud, 2016;14(2): 98-105.

3. Gonzalez $\mathrm{G}$, Resistencia a Insecticidas en el mosquito vector del dengue Aedes Aegypti (L) en sus epocas de transmisión de la enfermedad en Mérida, Yucatán [tesis doctoral], Mexico: Facultad de Ciencias Biológicas , Universidad Autónoma de Nuevo León; 2013.

4. Fernández-Salas I. Biología y Control de Aedes aegypti. Manual de Operaciones.Editorial UANL. Monterrey, México. 1999.

5. Mesa A, Alvarado G, Licona N, Ramos Raguilar M. Residualidad del Temefos en depósitos domésticos y su efectividad en el control de larvas de Aedes aegypti en Honduras, MEDISAN. 2013; 17(6):934-43

6. Palma H, Cabrera R, MoscosoM. Factores detrás de la renuencia al control vectorial del dengue en tres distritos del norte del Perú. Rev Peru Med Exp Salud Publica. 2016;33(1):13-20.

7. Rodriguez M, Bisset J, Fernandez Ditter, Perez O. Resistencia a insecticidas en larvas y adultos de Aedes aegypti: prevalencia de la esterasa A4 asociada con la resistencia a temefos, Rev Cubana Med Trop. 2004;56(1):54-60.

8. Cabezas C, Fiestas V, García-Mendoza M, Palomino M, Mamani E, Donaires F.Dengue en el Perú:a un cuarto de siglo de su reemergencia. Rev. Perú Med Exp Salud Publica.2015;32(1):146-56.

9. Rodríguez M, Terán M, Lazcano J, Leyva Y, Pacheco L, López I. Eficacia de Pyriproxyfeno en cepas de referencia de Aedes aegypti suceptible y resistente a temefos. Revista cubana de Medicina Tropical. 2014;65(3):339-49.

10. Palomino $M$, Solari L, León $W$, Vega $R$, Vergaray $M$, Cubillas L.Evaluación del efecto residual del temephos en larvas de Aedes Aegypti.Rev Perú Med Exp Salud Pública. 2006; 23(3): 158-62

11. Maestre R, Rey G, Salas J, VergaraC, Santacoloma L, Goenaga $\mathrm{S}$, et al. Estado de suceptibilidad de Aedes aegypti a 
insecticidas en Atlántico (Colombia). Revista Colombiana de Entomología. 2010;36(2);242-8

12. Griñan L. Protocolo para pruebas de Laboratorio y composicion larvicidas de mosquitos. Tecnologia Quimica y Comercio SA.

13. World Health Organization. Guidelines for Laboratory and Field Testing of Mosquito Larvicides. Document. Switzerland: World Health Organization; 2005.

14. Organización Mundial de la Salud. Instructions for determining the susceptibility or resistance of mosquito larvae to insecticides. Geneva: WHO; 1981. p. 6

15. Camargo S. Organization and administration of Aedesaegypt control and eradication programmes. Bull World Health Organ. 1967; 36(4): 610-3.

16. Rodríguez R. Estrategias para el control del dengue y del Aedes aegypti en las Américas. Rev Cubana MedTrop. 2002; 54(3): 189-201.

17. Reiner E. Spontaneous reactivation of phosphorylated and carbamylated cholinesterases. Bull World Health Organ. 1971; 44(1-2-3): 109-12.

18. Sharma S, Saxena V, La I. Study on susceptibility status in aquatic and adult stages of Aedes aegypti and Aedes albopictus against insecticides at international airports of South India. J Commun Dis. 2004; 36(3):177-81.

19. Chávez J, Córdova O, Vargas F. Niveles de susceptibilidad a temephos en el vector transmisor del dengue en Trujillo, Perú, Anales de la Facultad de Medicina Universidad Nacional Mayor de San Marcos. 2005; 66(1):53-6
Fuentes de Financiamiento

Este artículo ha sido financiado por la autora.

\section{Conflicto de interés}

La autora declara no tener ningún conflicto de interés.

\section{Correspondencia:}

María Elizabeth Pérez Alarcón

Dirección: Av. El corregidor 1531 Las Viñas La Molina.

Teléfono: 938101886

Correo electrónico: alarconp104@hotmail.com

Recibido: 26 de julio de 2017

Evaluado: 01 de agosto de 2017

Aprobado: 13 de setiembre de 2017

(c) La revista. Publicado por Universidad de San Martín de Porres, Perú. (c) в bajo términos de Licencia Creative Commons Atribución 4.0 Internacional. (http://creativecommons.org/licenses/by/4.0/) 